\title{
Shape and kinematics of elliptical galaxies: evolution due to merging at $z<1.5$
}

\author{
A. C. González-García, J. Oñorbe, R. Domínguez-Tenreiro, and M. Á. Gómez-Flechoso
}

\author{
Departamento de Física Teórica, Universidad Autónoma de Madrid, 28049 Madrid, Spain \\ e-mail: c.gonzalezgarcia@uam.es
}

Received 1 December 2008 / Accepted 14 January 2009

\begin{abstract}
Aims. We investigate the evolution in the shape and kinematics of elliptical galaxies in a cosmological framework. Methods. We identified relaxed, elliptical-like objects (ELOs) at redshifts $z=0, z=0.5, z=1$ and $z=1.5$ within a set of hydrodynamic, self-consistent simulations completed for a concordance cosmological model.

Results. The population of elliptical systems that we analysed evolve systematically with time becoming rounder in general by $z=0$ and also more velocity dispersion supported. We found that this is due primarily to major dry mergers where only a modest amount of angular momentum is involved in the merger event. Despite the general trend, in a significant number of cases the merger event involves a relatively high amount of specific angular momentum, which causes the system in general to acquire higher rotational support and/or a more oblate shape. These evolutionary patterns persist when we study our systems in projection, in simulating true observations, and thus should be evident in future observations.
\end{abstract}

Key words. galaxies: elliptical and lenticular, $\mathrm{cD}$ - galaxies: fundamental parameters - galaxies: interactions - galaxies: evolution galaxies: formation - Galaxy: kinematics and dynamics

\section{Introduction}

Surveys of high-redshift galaxies suggest that some relaxed, massive elliptical galaxies may already be in place at $z \sim 2-1.5$ (Cimatti et al. 2004; Conselice et al. 2007), or even by $z \sim 5$ (Wiklind et al. 2008). These results notwithstanding, according to current formation scenarios, merging has played an important role in the mass assembly of most of the local massive elliptical galaxies. Observations and theory suggest that, first, violent mergers at high $z$ have transformed most of the available gas into stars, and, later on, these systems might have evolved through gas-free (the so-called dry) mergers (Conselice 2006; see otherwise Scarlata et al. 2007).

Since the study by Bertola \& Cappacioli (1975), we know that elliptical galaxies are supported against gravity by random motions as well as rotation. Davies et al. (1983) studied the now classical $V_{\max } / \sigma_{\mathrm{o}}$ versus $\epsilon$ diagram for spheroids (Illingworth 1977; Binney 1978), where $V_{\max }$ is the maximum of the lineof-sight (1.o.s.) rotation curve, $\sigma_{\mathrm{o}}$ is the central 1.o.s. velocity dispersion of the galaxy, and $\epsilon$ is the mean ellipticity inside a given radius. They found that luminous (and massive) elliptical galaxies were characterised by low $V_{\max } / \sigma_{\mathrm{o}}$ and a fairly circular morphology (low $\epsilon$ ), while ellipticals with intermediate luminosity tend to have higher values of both $V_{\max } / \sigma_{\mathrm{o}}$ and $\epsilon$. Several observations involving 1D spectroscopy of near by elliptical galaxies (Lauer 1985; Bender 1988; Nieto et al. 1989; Bender et al. 1994; Pellegrini 2005; Lauer et al. 2005), and 2D spectroscopy confirmed this characteristics for ellipticals (see Emsellem et al. 2007; and Cappellari et al. 2007, hereafter CAP07). Van der Marel \& van Dokkum (2007) presented evidence of evolution in the rotation support of elliptical systems since $z=0.5$. Present formation schemes should be capable of explaining these observations of the kinematics and shapes of elliptical galaxies and their possible evolution.

A number of $\mathrm{N}$-body simulations of isolated galaxy mergers have dealt with the population of the classical diagram and the formation of boxy and disky objects (Naab \& Burkert 2003; González-García \& Balcells 2005; González-García \& van Albada 2005; Naab et al. 2006; Bournaud et al. 2005; Robertson et al. 2006; Cox et al. 2006; González-García et al. 2006; Jesseit et al. 2007; Naab \& Ostriker 2007). These studies indicate that mergers between disk galaxies tend to produce too high rotational support when compared with present-day massive elliptical galaxies. Mergers between elliptical galaxies have indeed been shown to reproduce the observed characteristics of massive ellipticals. Khochfar \& Burkert (2003) and Kang et al. (2007, and references therein) presented the first attempts by semi-analytical modelling to recreate the kinematics and shapes of early-type galaxies. Naab et al. (2007) studied the formation of three massive galaxies from cosmological initial conditions. However, a detailed, statistical analysis of the internal kinematics and shapes of objects formed in fully self-consistent cosmological simulations and their possible evolution is, to the best of our knowledge, still missing yet mandatory in providing a clear picture of the mechanisms at play in the formation and evolution of present day Ellipticals (E's).

In this paper, we present the results of self-consistent cosmological simulations that we developed to investigate the shape and kinematical evolution of elliptical-like objects (hereafter ELOs) at several redshifts. In the present analysis, we consider both three-dimensional (hereafter, 3D) and projected data on the sky. Other kinematic parameters proposed will be investigated in a forthcoming paper, and are omitted from the present study for the sake of simplicity. 


\section{Methods and numerical experiments}

\subsection{Simulations}

We completed seven hydrodynamical simulations in the context of a concordance cosmological model (Spergel et al. 2006). For five of the simulations, we employed a $10 \mathrm{Mpc}$ side periodic box with flat $\Lambda$ CDM cosmological model, with $h=0.65, \Omega_{\mathrm{m}}=$ 0.35 and $\Omega_{\mathrm{b}}=0.06$. To set the initial conditions, we employed the algorithm developed by Couchman (1991) with a slightly high normalisation parameter input $\sigma_{8}=1.18$, compared to the average fluctuations, to mimic an active region of the Universe (Evrard et al. 1990). These simulations represented the A-sample in Oñorbe et al. $(2005,2006,2007)$ and they differ from each other in the seed used to generate the initial conditions.

To test whether the evolutionary trends found in these simulations are robust, we executed two simulations with a box side twice as long as in the first simulations and a factor of 8 higher number of particles, for the same cosmology as before, now using input values $\sigma_{8}=0.75$ and 0.95 . The first of these values is such that the initial distribution of kinetic energy per unit volume is similar to that in the small box simulations (Gelb \& Bertschinger 1994; Sirko 2005; Power \& Knebe 2006). To execute the small and larger box simulations, we used respectively DEVA (Serna et al. 2003) and P-DEVA (its OpenMP version, see Serna et al., in preparation), which are two Lagrangian SPH-AP3M codes. The mass resolution is $1.28 \times 10^{8} M_{\odot}$ for dark-matter particles and $2.67 \times 10^{7} M_{\odot}$ for baryon particles. Star-formation (SF) processes were included in terms of a simple phenomenological parametrisation (Katz 1992; see also Serna et al. 2003 and Oñorbe et al. 2007, for details).

We applied a consistency test by executing two simulations with identical initial conditions in a $10 \mathrm{Mpc}$ side periodic box, one containing $2 \times 64^{3}$ particles and a second containing $2 \times$ $128^{3}$ particles, with masses as above for the $2 \times 64^{3}$ simulation and $1.6 \times 10^{7} M_{\odot}$ for dark-matter particles and $3.3 \times 10^{6} M_{\odot}$ for baryon particles in the $2 \times 128^{3}$ simulation.

Galaxy-like objects of different morphologies appear in the simulations. ELOs were identified as those having a prominent, relaxed spheroidal component, consisting of stars, with no extended discs. These baryonic objects are embedded in a dark matter extended halo, typically 10 times more massive. In Oñorbe et al. (2005, 2006), it was shown that these ELOs satisfy dynamical Fundamental Plane relations. In Oñorbe et al. (2007), the total, bright, and dark matter profiles of ELOs and their kinematics were analysed and found to be in satisfactory agreement with observational data.

\subsection{Methods and analyses}

We analysed the ELOs at four redshifts: $z=1.5, z=1.0, z=0.5$, and $z=0$. We selected undisturbed ELOs (i.e. within their limiting radius, we observe a quasi-equilibrium behaviour of the system) because we wanted to analyse their shapes, although their dark matter halos could be in the first stages of merging. We analysed those systems that at each redshift have more total stellar mass than $2.6 \times 10^{10} M_{\odot}$. This limit agrees with Kauffmann et al. (2003) for early-type galaxies, and the number of particles per ELO is sufficiently large to avoid resolution problems (see below). In total, we have 425 ELOs ( 225 from the small box simulations, and 200 from the large box simulations), 127 at $z=0,107$ at $z=0.5,97$ at $z=1.0$, and 94 at $z=1.5$.

To quantify the shape of the ELO, we measured the axis ratios ( $c / a$ versus $b / a$, with $a>b>c)$ of the ellipsoidal figures defined by the particles inside $r_{90}$ (the radius enclosing $90 \%$ of the stellar mass of the system), by computing the eigenvalues of their inertia tensor (see González-García \& van Albada 2005). To classify the shape of our objects, we used the triaxiality parameter introduced by de Zeeuw \& Franx (1991), $T=\left(1-(b / a)^{2}\right) /\left(1-(c / a)^{2}\right)$, in the following way: we introduce the parameter $s$ as $s=3$ if $c / a>0.9$ (close to spherical objects), $s=2$ if $c / a<0.9$ and $T<0.3$ (oblate objects), $s=0$ if $c / a<0.9$ and $T>0.7$ (prolate objects), and $s=1$ elsewhere (triaxial objects). Concerning kinematics, we studied the three dimensional velocity dispersion inside the effective radius $r_{\mathrm{e}}{ }^{1}$ $\left(\sigma_{3 \mathrm{D}}\right)$, and considered the mean tangential velocity at $r=r_{\mathrm{e}}$, $\left(V_{\phi}\right)$. We also studied these characteristics at $r_{90}$.

To compare with classical observations, we investigated the line-of-sight rotation and velocity dispersion measurements to one projected effective radius $\left(R_{\mathrm{e}}\right)$ for each object. We chose a point of view perpendicular to the spin angular momentum vector of the stellar matter because these particular points of view should maximise the effects of rotation when evident (see Binney 2005; Burkert \& Naab 2005). We first derived the ellipticity of each ELO by projecting the complete particle distribution onto a plane perpendicular to the line-of-sight. Local surface densities were then computed following a logarithmic binning of space. Ellipses were fitted to the ELO isophotes, as performed for observational data. The ellipticity computed in our analysis was taken to be the mean of the ellipticities inside one $R_{\mathrm{e}}$. Although the number of particles may not be very high in some objects, the signal at half the mass radii in projection is sufficiently high to calculate these values. We performed consistency tests with methods based on a diagonalization of the momentof-inertia tensors, and values were consistent with each other. To derive the line-of-sight rotation and velocity dispersion profiles, we placed a slit along the major axis of the projected system (as obtained in the previous step) and projected the velocities of each particle along the line of sight. From these curves, we finally derived the central l.o.s. velocity dispersion $\left(\sigma_{\mathrm{o}}\right)$ and the maximum of the velocity curve inside $R_{\mathrm{e}}\left(V_{\max }\right)$.

To understand the physical processes underlying the shape and rotation-support changes, for each of the 127 ELOs at $z=0$ we compiled its mass aggregation track (MAT) along the main branch of its merger tree, for both its baryonic (the mass inside fixed radii) and total mass (the virial mass). These MATs provide information about the mass assembly processes with time. Major mergers (MM; $\left.M_{\text {secondary }} / M_{\text {primary }}>0.25\right)$, minor mergers (mM), and aggregation (i.e. smooth in-fall of mostly gaseous material) processes can be clearly identified. We can also compute the amount of dissipation involved in the different processes as well as estimate the amount of angular momentum involved. Complementary information about a merger is provided by the configurations shown, in a time interval around the merger event, by the baryonic particles destined to form the ELO later on, i.e. the type and number of objects involved, and the environment. This way we analysed 150 events of any type along the main branch of the merger tree at $z<1.5$, and classified them into several categories: $\mathrm{mM}$ or MM; binary or multiple; or involving a high or a low amount of specific angular momentum (and their combinations).

\subsection{Consistency checks}

Figure 1 shows the results of the consistency simulations. Figure 1 (left panel) shows the star-formation history of two

${ }^{1} r_{\mathrm{e}}$ is the radius enclosing half of the stellar mass of the system. 

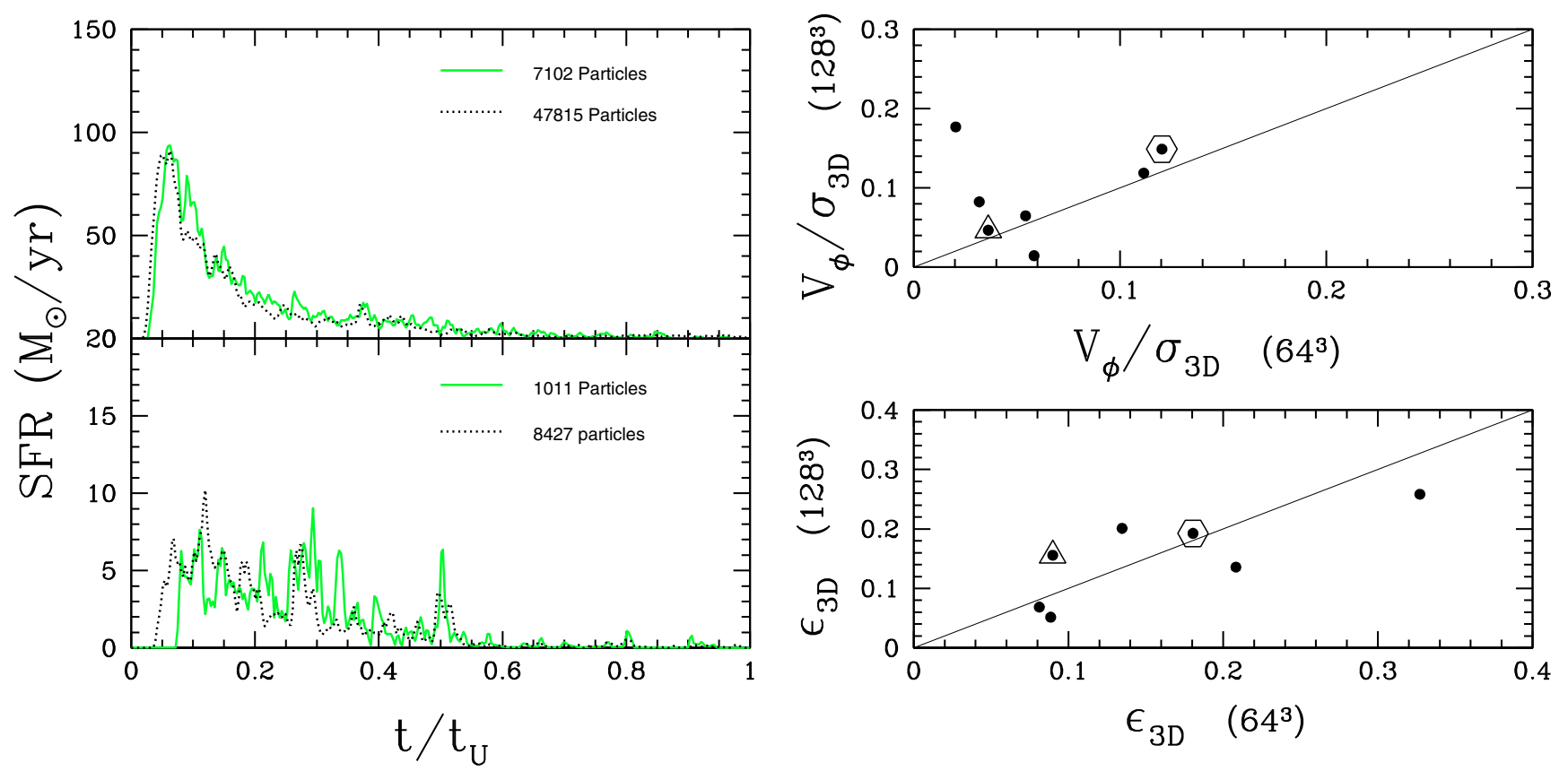

Fig. 1. Left: star formation history of the most (top) and least (bottom) massive ELOs for the test simulations with $2 \times 64^{3}$ particles (solid green line) and $2 \times 128^{3}$ particles (dotted black line). Right, comparison of the kinematic (top) and shape (bottom) observables. The most massive system is the marked with a triangle and the least massive with an hexagon. For details, see text.

objects, the most and least massive ELOs in the simulations. The black dotted line and the solid green line depict the results of the simulations with more and less particles, respectively. We found small differences, especially at early times, although the two systems display a general similar behaviour, and at high cosmic times (low redshift) no significant differences are evident. A similar test was completed by Naab et al. (2007) and, although the numerical approaches differ, it is reassuring to also find convergence in this resolution test.

Figure 1 (right panel) presents comparative results of the same objects at $z=0$ in the two simulations where we computed the observables introduced above on shape and kinematics. The systems appear to be stable and agreement between the simulations results is good, although one object does exhibit a significant difference. This system is not the least massive in these simulations and the difference is due to the peculiar way in which the $V_{\phi}$ parameter is measured, such that particles at different radii are considered. The overall consistency in these tests prompt us to use the $2 \times 64^{3}$ simulations for the sake of computing time.

\section{Results}

\subsection{Shape and kinematic evolution}

Figure 2, top row, shows the shape for the 4 redshifts, presented in different colours. Figure 2 a shows $c / a$ versus $b / a$. Blue symbols $(z>1)$ appear mostly in the middle-left of the diagram, while red symbols $(z=0)$ appear in the top right, that is, the values of $c / a$ and $b / a$ tend to increase with decreasing redshift, or in summary, the population of ELOs become rounder on average with time. These results hold when we use $r_{\mathrm{e}}$ instead of $r_{90}$ to calculate inertia tensors, although in this case ELOs tend to appear even rounder. Figure $2 b$ shows a histogram for the shape parameter $s$ defined above. Results are given in relative numbers, and the error bars express the Poissonian noise. Most of our systems are triaxial objects at all redshifts, except at $z>1$ when there is a fair amount of prolate systems. For all the simulations we consider here, the fraction of prolate objects decreases with decreasing redshift, and at all redshifts there is a small number of perfect spheres. The number of triaxial and oblate objects also increases with decreasing redshift. It is noteworthy that our results on ELO shapes at $z=0$ compare reasonably well with those obtained from the Sloan survey for elliptical galaxies (Kimm \& Yi 2007), where they show that the sample consists of about $45 \%$ triaxial objects and around 26-29\% prolate and oblate objects.

Figure 2c, illustrates the rotational support of the ELOs. The histograms are normalised to their total number at each redshift, and the error bars represent the Poissonian noise. There is a trend towards increasing the number of systems with a lower value of $V_{\phi} / \sigma_{3 \mathrm{D}}$ as $z$ decreases; this trend appears independently of the details of the simulation. Figure $2 \mathrm{~d}$ shows the diagram of $V_{\phi} / \sigma_{3 \mathrm{D}}$ versus $\epsilon_{3 \mathrm{D}}$, where $\epsilon_{3 \mathrm{D}}=1-c / a$. Blue objects $(z>1)$ appear mostly again at high $\epsilon_{3 \mathrm{D}}$ values with a broad range of $V_{\phi} / \sigma_{3 \mathrm{D}}$ values, while at $z=0$ (red symbols), $\epsilon_{3 \mathrm{D}}$ and $V_{\phi} / \sigma_{3 \mathrm{D}}$ are smaller on average. These trends persist if we use $r_{90}$ instead of $r_{\mathrm{e}}$ (i.e., we measure external rotation instead of rotational support), although in this case there is a higher fraction of systems with higher values of $V_{\phi} / \sigma_{3 \mathrm{D}}$.

We performed Kolmogorov-Smirnov tests to check whether the samples at different $z$ 's originate for the same distribution. Such null hypotheses can be ruled out in all cases, both for shapes and rotational support, at confidence levels of between 95 and $99.9 \%$. Given the consistency in the direction of the more frequent changes (i.e., towards becoming "rounder" and more pressure-supported), we refer to these changes as an evolutionary track. We emphasise that this evolution concerns the global population of E's rather than individual ones.

We now consider the 2D analysis. First of all, there is reasonable agreement between the 3D and the projected kinematics, i.e. the rapidly rotating systems are in agreement in both samples. Also, objects with higher values of $c / a(\sim 1)$ tend to be those with the smaller $\epsilon$, while the larger ellipticities are found 

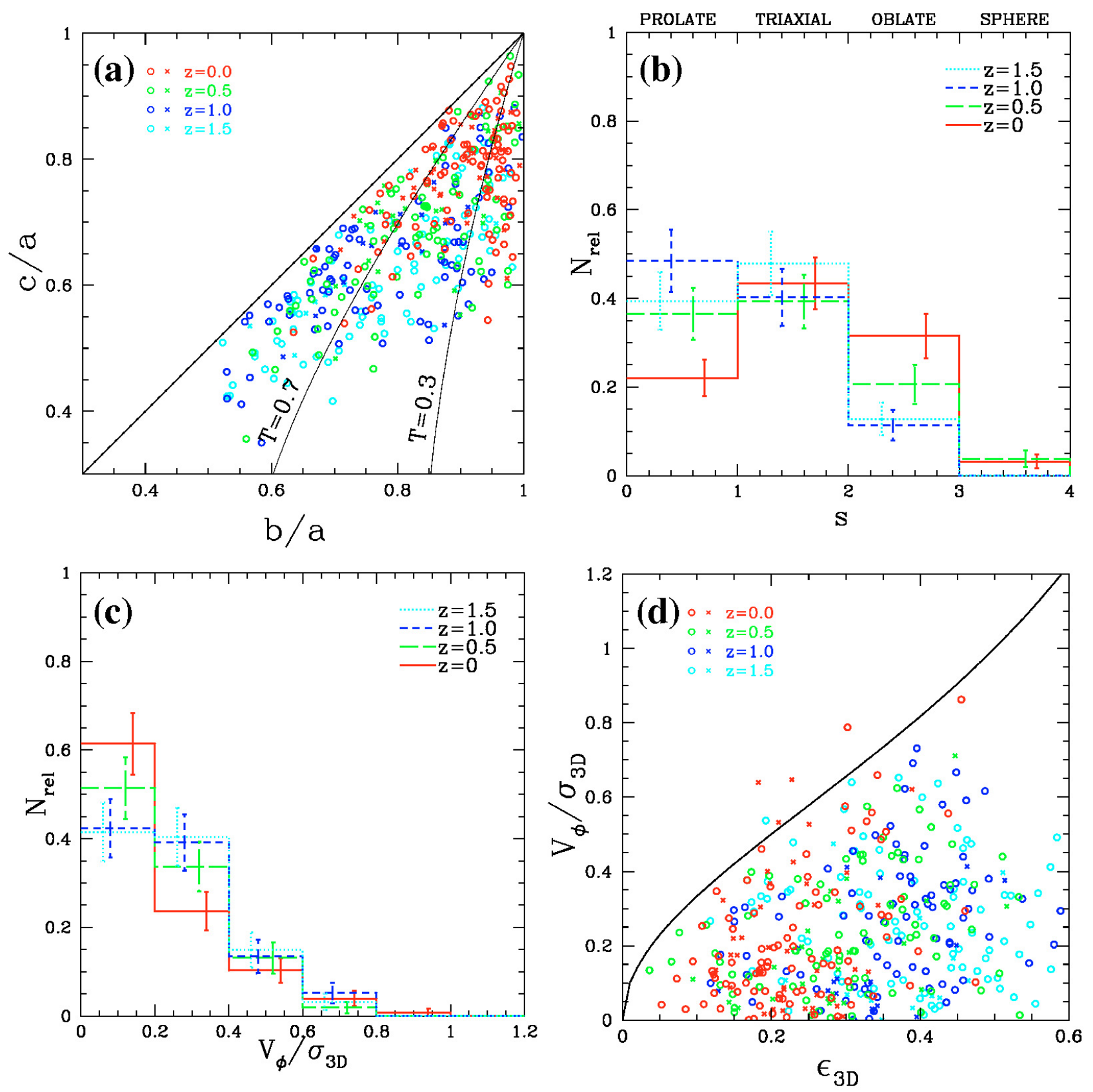

Fig. 2. a) Axis ratios of the spheroids. Colours indicate the different redshits. Crosses are objects more massive than $M_{*}>1 \times 10^{11} M_{\odot}$, circles are objects of lower mass. b) Histograms of the shape parameter $S$ vs. relative number for all our systems. Different lines and colours indicate the histograms for the four redshift bins. c) Histograms of the rotational support measured as $V_{\phi} / \sigma_{3 \mathrm{D}}\left(\right.$ at $\left.r_{\mathrm{e}}\right)$ in relative number for our systems, colours and lines as in b). d) $V_{\phi} / \sigma_{3 \mathrm{D}}$ vs. $\epsilon_{3 \mathrm{D}}$ for the ELOs, colours and symbols as in a) panel. Black solid line indicates the locus for oblate rotators. For details, see text.

among those objects with lower $c / a$. This correlation between $3 \mathrm{D}$ and $2 \mathrm{D}$ results for ELOs is noteworthy because it implies that the intrinsic evolution detected in virtual systems, should also be detected in observational (projected) data, if it occurs in true elliptical galaxies.

Figure 3 presents the results for the classical $V_{\max } / \sigma_{\mathrm{o}}$ versus $\epsilon$ diagram (Davies et al. 1983). For the sake of clarity, we plot only the most massive objects $M_{*}>1 \times 10^{11} M_{\odot}$ of the smallsized simulations and the values are measured from a point of view perpendicular to the angular momentum vector. Each object is indicated by an open symbol, the colour representing the redshift of that object. At high $z$ (light and dark blue), ELOs tend to appear as more flattened systems with a high relative rotation.
However, at lower redshifts (green and red), we have more massive systems, which tend to appear rounder and more pressuresupported (except those few with high $V_{\phi} / \sigma_{3 \mathrm{D}}$ ), in agreement with the 3D data (see Fig. 2d). The filled circles indicate the mean of the distribution at each redshift and the error bars are a measure of the standard deviation in the distribution. The systems evolve on average from being flat and relatively rotationally supported to being rounder/triaxial and pressure-supported systems. Finally, we note that if we include all systems in this plot, the evolution still holds. The size of the open symbols in Fig. 3 is a measure of the accumulated number of major mergers that the system has undergone since $z=1.5$. Larger symbols are mostly located in the lower left part of the diagram. 


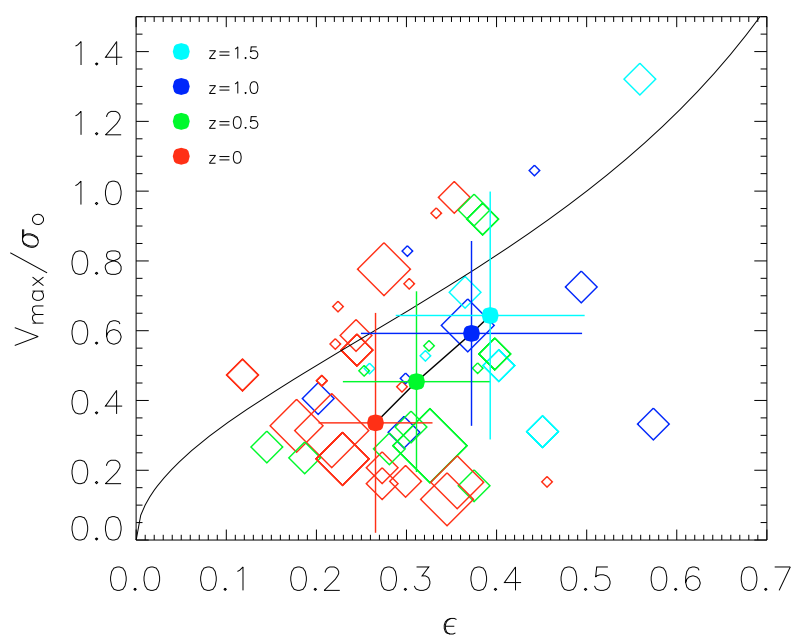

Fig. 3. $V_{\max } / \sigma_{\mathrm{o}}$ vs. $\epsilon$ diagram. The black solid curve gives the locus of the oblate rotators (Binney 1978). Only models with $M_{*}>1 \times 10^{11} M_{\odot}$ are plotted. Colours are as in Fig. 2. Filled circles give a mean for the objects at each redshift. Finally the size of the symbol gives the accumulated number of major mergers that a system has undergone.

This indicates that merging is one of the key ingredients driving this evolution towards rounder and more pressure-supported ELOs. Although this is the general trend, we also note that there are some cases with high $V_{\max } / \sigma_{\mathrm{o}}$ and a significant number of mergers, and this indicates that merging effects could be far more complex.

It has been argued (Binney 2005; Burkert \& Naab 2005; CAP07) that the $V_{\max } / \sigma_{\mathrm{o}}$ versus $\epsilon$ diagram is affected by projection effects. The results presented in Fig. 3 are obtained by looking at each object from a point of view perpendicular to the angular momentum vector. For oblate rotators, this vector should be close to the short principal axis, and the projected image would provide the maximum ellipticity and $V_{\max } / \sigma_{0}$. However, many of our most massive systems are triaxial and the angular momentum vector has neither to coincide or be close to the short principal axis. In these cases, the $V_{\max } / \sigma_{\mathrm{o}}$ diagram could be affected by projection effects. To test the effects of projection we have analysed the systems along 100 randomly chosen lines of sight, deriving the projected observables of the classical diagram. We obtained a distribution of points, which in the case of the most massive ELOs did not differ significantly from the points presented in Fig. 3. We tested the null hypothesis that the distributions of points at the different redshifts originate in the same distribution by completing a Kolmogorov-Smirnov test. The result is that we can exclude this hypothesis at the $99.9 \%$ confidence level. The same result is obtained by a Kuiper test. We conclude that the results presented are robust and that the evolution observed in the projected quantities in shape and kinematics is indeed real.

\subsection{Merging rate}

To understand how merging affects evolution, we carefully studied and classified all the merger events along the MATs of the 127 ELOs identified at $z=0$.

We investigated the number of mergers in three redshift intervals $z \in(1.5,1) ;(1,0.5)$ and $(0.5,0)$. We were particularly interested in the mergers experienced by our ELOs. We investigated the main branch of the ELO merger tree and looked at the mass aggregation track to identify increments in mass. We

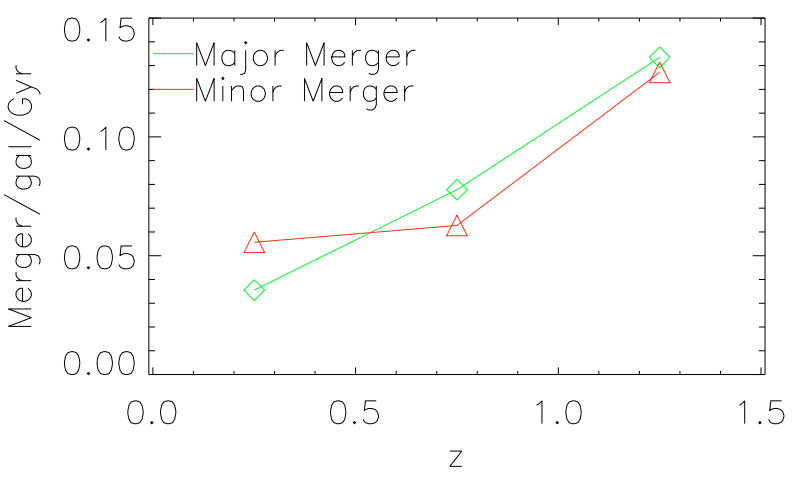

Fig. 4. Number of mergers per galaxy per Gyr as a function of redshift interval. Red line gives the rate for minor mergers. Green line gives the rate for major mergers. We observe a decline in the number of mergers, this decline is slightly steeper in the fraction of MM.

identified major mergers $(\mathrm{MM})$ and minor mergers $(\mathrm{mM})$. For the three redshift intervals, we identified 21, 23, and $23 \mathrm{MMs}$, and 20, 22, and $36 \mathrm{mMs}$. Figure 4 shows the evolution in the merger rate since $z=1.5$ for the three redshift bins. The merger rate is expressed in terms of the number of mergers per galaxy per gigayear. We measured a decrease in the number of mergers, which is more pronounced in the MM fraction than in $\mathrm{mM}$.

We note that this result concerns the number of mergers experienced by the ELOs in our sample only along the main branch of their merger tree. Also, we analysed the high-mass end of the galaxy mass distribution, not taking into account mergers resulting in disk-like systems or systems of smaller mass. Despite these caveats, we compared these numbers with recent theoretical results. Naab et al. (2007) performed cosmological simulations including gas, cooling, and star formation to investigate the formation of three systems, two resembling true elliptical galaxies and a third resembling an S0 galaxy. They found that at $z<1$, the systems have experienced few merger events, with one major merger in one case and a minor merger in another one. Scannapieco et al. (2008) used results from the Aquarius simulations (Springel et al. 2008) to investigate the survival of disks. The number of mergers is an important aspect in estimating the impact of merging in the survival of disks. They found that the disks have suffered a moderate number of mergers since $z=2$. Finally, using the Millennium simulation Genel et al. $(2008,2009)$ found that since $z=2.2$, the number of MM experienced by a halo of mass $M$ in the redshift interval $z_{i}$ and $z_{f}$ can be approximated by $\bar{N}_{\mathrm{MM}}\left(z_{i}, z_{f}, M\right) \approx 0.13\left\{\log \left[M /\left(10^{10} M_{\odot}\right)\right]+1\right\}\left(z_{i}-z_{f}\right)$. Our simulations predict slightly higher values of this parameter, perhaps related to the active environment conditions modelled in our runs. However, we found that the results are in agreement with Fig. 8 of Genel et al. (2009) and our numbers are consistent with these theoretical works. Finally, Conselice et al. (2009) studied the merger history of a large number of galaxies for $z<1.2$. Our results here are again in good agreement with those presented in their Fig. 7 for observational results.

\subsection{Trends of merger characteristics with time}

At high $z(z>1.5)$, dissipative, multiple MMs are more frequent than other merger types. At $z<1.5$, most mergers are rather dry (ELOs are mostly devoid of gas, with percentages of less than a few percent), with a higher frequency of low angular-momentum MM compared to high angular-momentum MMs (a frequency of $2: 1$ ). Most mergers involving a fair amount of specific 
angular momentum are multiple events. However, the opposite is not true, since there are multiple events where only a small amount of angular momentum is involved, and particularly so at $z>1.5$.

\section{Discussion}

We have compared the merger characteristics with the changes measured in shape and rotational support. We have found that there is a close correlation between the shape and the amount of rotational support of a given ELO at a given time and the characteristics of the last merger event it has suffered. Specifically, MMs always produce changes in both shape and rotational support in the outer and the inner parts. MMs with little angular momentum most often decrease the rotational support inside $r_{\mathrm{e}}$, producing mostly rounder (i.e., a larger value of $c / a$ ) prolate spheroids, while MMs with a high (intermediate) amount of angular momentum increase rotational support, producing oblate or triaxial systems. This picture of the role of angular momentum is consistent with the transformation found in binary mergers of spheroidal systems by González-García \& van Albada (2005). mMs most often produce triaxial or oblate spheroids and increase the rotational support, although the effect is most likely to affect the outer parts of the ELO (see Balcells \& Quinn 1990; Eliche-Moral et al. 2006), except for penetrating mMs. Finally, aggregation processes are also important and may affect the shape and kinematics of the final object. We have found that around $85 \%$ of the changes in both shape and rotational support are associated with merging (in contrast to aggregation).

Combining these findings on the trends of merger characteristics with time and the different effects a merger causes according to these characteristics, we can understand that prolate ELOs are the most frequent at high $z$, and they are then transformed most often into rounder triaxial objects with less rotational support. An interesting conclusion from this study is that, due to dry merging, the most luminous (i.e. massive) ellipticals must be rounder and in general less rotationally supported. Indeed, this is what is found in observational data (see e.g., Davies et al. 1983).

The results presented here are not intended to represent fully at a quantitative level the characteristics of the shape and kinematic evolution of elliptical galaxies, but rather to unveil some qualitative trends in dense environments and the mechanisms causing them in a cosmological context. It is reassuring to find that all the simulations analysed here show in general an evolution in the population of E's towards roundish and less rotationally supported systems, mostly driven by dry merging, and that the mechanisms at play described above, which cause either the general trend or the exceptions, are consistent in all kind of simulations, of different box size, particle number, input $\sigma_{8}$, and code.

Van der Marel \& van Dokkum (2007) reported a similar evolution for two samples of $\sim 40$ galaxies at $z=0.5$ and $z=0$. Although the evolution that we observe in our simulations seems milder than the one reported by their study, the amount of evolution remains unclear to higher redshifts. Thus, it would be highly desirable to perform larger statistical studies of the shape and kinematics of true elliptical galaxies at low $z$, and acquire observations of galaxy kinematics at higher redshifts to confirm the evolutionary trend of ellipticals predicted here.

Acknowledgements. We would like to thank the anonymous referee for stimulating comments that improved the quality of the paper. We thank A. Serna and
F. Martínez-Serrano for making available the new P-DEVA code for us. This work was partially supported by the MCyT (Spain) grants: AYA-07468-C03-03 and AYA2006-15492-C03-01 from the PNAyA, and the regional government of Madrid through the ASTROCAM Astrophysics network (S0505/ESP0237). We thank the Centro de Computación Científica (UAM, Spain) for computing facilities.

\section{References}

Balcells, M., \& Quinn, P. J. 1990, ApJ, 361, 381

Bender, R. 1988, A\&A, 193, L7

Bender, R., Saglia, R. P., \& Gerhard, O. E. 1994, MNRAS, 269, 785

Bertola, F., \& Capaccioli, M. 1975, ApJ, 200, 439

Binney, J. 1978, MNRAS, 183, 501

Binney, J. 2005, MNRAS, 363, 937

Bournaud, F., Jog, C. J., \& Combes, F. 2005, A\&A, 437, 69

Burkert, A., \& Naab, T. 2005, MNRAS, 363, 597

Cappellari, M., Emsellem, E., Bacon, R., et al. 2007, MNRAS, 379, 418 (CAP07)

Cimatti, A., Daddi, E., Renzini, A., et al. 2004, Nature, 430, 184

Conselice, C. J. 2006, ApJ, 638, 686

Conselice, C. J., Bundy, K., Trujillo, I., et al. 2007, MNRAS, 381, 962

Conselice, C. J., Yang, C., \& Bluck, F. L. 2009, MNRAS, submitted [arXiv: 0812.3237v1]

Couchman, H. M. P. 1991, ApJ, 368, L23

Cox, T. J., Dutta, S. N., Di Matteo, T., et al. 2006, ApJ, 650, 791

Davies, R. L., Efstathiou, G., Fall, S. M., Illinwort, G., \& Schechter, P. L. 1983, ApJ, 266, 41

de Zeeuw, T., \& Franx, M. 1991, ARA\&A, 29, 239

Eliche-Moral, M. C., Balcells, M., Aguerri, J. A. L., \& González-García, A. C. 2006, A\&A, 457, 91

Emsellem, E., Cappellari, M., Krajnović, D., et al. 2007, MNRAS, 379, 401

Evrard, A., Silk, J., \& Szalay, A. S. 1990, ApJ, 365, 13

Genel, S., Genzel, R., Bouché, N., et al. 2008, ApJ, 688, 789

Genel, S., Genzel, R., Bouché, N., Naab, T., \& Sternberg, A. 2009, ApJ, submitted [arXiv:0812.3154v1]

Gelb, J. M., \& Bertschinger, E. 1994, ApJ, 436, 467

González-García, A. C., \& Balcells, M. 2005, MNRAS, 357, 753

González-García, A. C., \& van Albada, T. S. 2005, MNRAS, 361, 1030

González-García, A. C., Balcells, M., \& Olshevsky, V. S. 2006, MNRAS, 372, L78

Illingworth, G. 1977, ApJ, 218, L43

Jesseit, R., Naab, T., Peletier, R. F., \& Burkert, A. 2007, MNRAS, 376, 997

Kang, X., van den Bosch, F. C., \& Pasquali, A. 2007, MNRAS, 381, 389

Katz, N. 1992, ApJ, 391, 502

Kauffmann, G., Heckman, T. M., White, S. D. M., et al. 2003, MNRAS, 341, 33

Khochfar, S., \& Burkert, A. 2003, ApJ, 597, 117

Kimm, T., \& Yi, T. S. 2007, ApJ, 670, 1048

Lauer, T. R. 1985, MNRAS, 216, 429

Lauer, T. R., Faber, S. M., Gebhardt, K., et al. 2005, AJ, 129, 2138

Naab, T., \& Burkert, A. 2003, ApJ, 597, 893

Naab, T., \& Ostriker, J. P. 2007 [arXiv: astro.ph/0702535]

Naab, T., Khochfar, S., \& Burkert, A. 2006, ApJ, 636, L81

Naab, T., Johansson, P. H., Ostriker, J. P., \& Efstathiou, G. 2007, ApJ, 658, 710

Nieto, J.-L., \& Bender, R. 1989, A\&A, 215, 266

Oñorbe, J., Domínguez-Tenreiro, R., Sáiz, A., Serna, A., \& Artal, H. 2005, ApJ, 632, L57

Oñorbe, J., Domínguez-Tenreiro, R., Sáiz, A., Artal, H., \& Serna, A. 2006, MNRAS, 373, 503

Oñorbe, J., Domínguez-Tenreiro, R., Sáiz, A., \& Serna, A. 2007, MNRAS, 376, 39

Power, C., \& Knebe, A. 2006, MNRAS, 370, 691

Pellegrini, S. 2005, MNRAS, 364, 169

Robertson, B., Cox, T. J., Hernquist, L., et al. 2006, ApJ, 641, 21

Scannapieco, S., White, S. D. M., Springel, V., \& Tissera, P. B. 2008, MNRAS, submitted [arXiv:0812.0976v1]

Scarlata, C., Carollo, C. M., Lilly, S. J., et al. 2007, ApJS, 172, 494

Serna, A., Domínguez-Tenreiro, R., \& Sáiz, A. 2003, ApJ, 597, 878

Sirko, E. 2005, ApJ, 634, 728

Spergel, D. N. 2006, IAUJD, 7, 6

Springel, V., Wang, J., Vogelsberger, M., et al. 2008, MNRAS, 391, 1685

van der Marel, R. P., \& van Dokkum, P. G. 2007, ApJ, 668, 738

Wiklind, T., Dickinson, M., Ferguson, H. C., et al. 2008, ApJ, 676, 781 